\title{
Screening of genes involved in cAMP-mediated signalling in a large Italian series of patients affected with Albright hereditary osteodystrophy and/or Pseudohypoparathyroidism
}

Elli FM, Bordogna P, deSanctis L, Spada A, Mantovani G

Dep. of Clinical Sciences and Community Health, University of Milan, Endocrinology and Diabetology Unit, Fondazione IRCCS Ca' Granda Ospedale Maggiore Policlinico

\section{INTRODUCTION}

Pseudohypoparathyroidism (PHP) is a heterogeneous group of rare genetic metabolic disorders due to molecular defects at the GNAS locus, that encodes also for the $\alpha$-subunit of the stimulatory G protein (Gsa), causing end-organ resistance to the actions of PTH. The classification of the different subtypes of PHP is based on the presence of specific somatic and developmental abnormalities, referred to as Albright hereditary osteodystrophy (AHO), and the resistance to other hormones acting via GPCRs.

Recently, mutations in genes encoding proteins crucial for cAMP-mediated signalling different from Gs $\alpha$ and deletions of chromosome 2 q37.2 have been detected in a small subset of patients with PHP with no GNAS defects, showing a phenotypic overlap with Acrodysostosis (ACRDYS) and brachydactyly-mental retardation syndrome (BDMR), also called AHO-like syndrome. Despite the high detection rate of genetic and epigenetic defects by currently available molecular approaches, about $30 \%$ of PHP patients still lack a molecular diagnosis, hence the need to screen patients negative for GNAS genetic or epigenetic defects also for chromosomal regions and genes associated to diseases that undergo differential diagnosis with PHP.

\section{MATERIALS \& METHODS}

In this study, we screened by Sanger sequencing and multiplex ligand-dependent probe amplification (MLPA) our series of AHO/PHP patients negative for GNAS locus genetic and imprinting defects (sporadic or genetic-based), for the presence of mutations at PRKAR1A gene ( $n=79)$, PDE4D gene ( $n=18)$, as well as for deletions affecting the subtelomeric chromosome region $2937(n=89)$.

\section{Sanger sequencing}

Genomic DNA was extracted by Nucleon BACC2 genomic DNA purification kit from peripheral blood leukocytes of both patients and parents (GE Healthcare, Piscataway, NJ, USA) according to the manufacturer's instructions. PRKAR1A and PDE4D exons and flanking intronic sequences were amplified by PCR and direct sequencing of amplicons was performed using the AmpliTaq BigDye Terminator kit and 3100 Genetic Analyzer (Applied Biosystens, Foster City, CA). The mutation nomenclature follows the guidelines indicated by Human Genome Variation Society (HGVS).

\section{PRKAR1A}

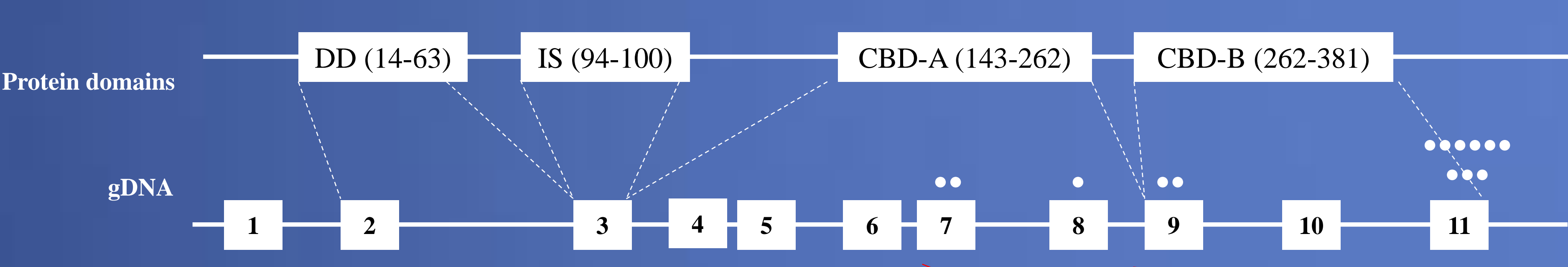

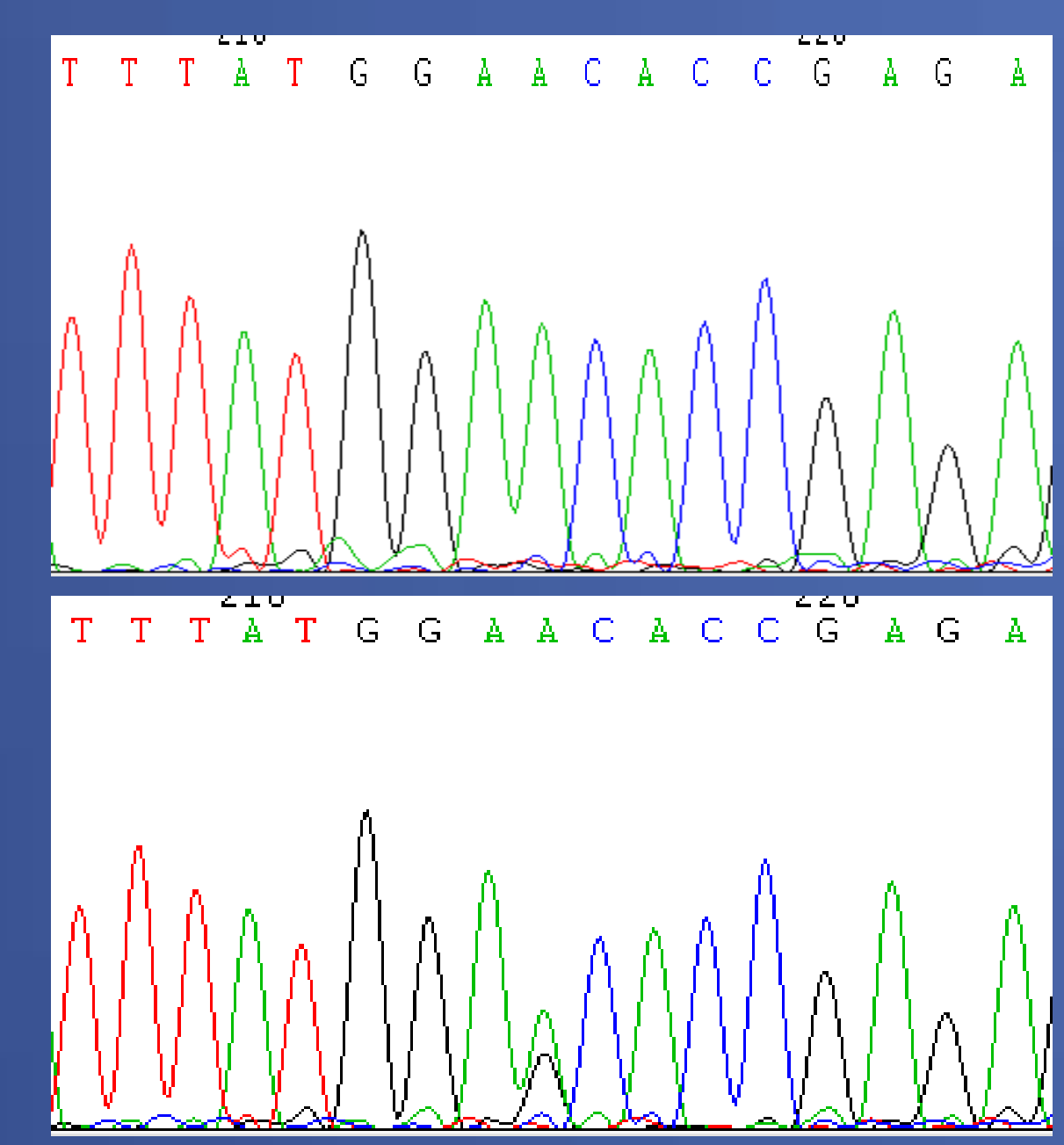

$\underset{c .625 \mathrm{~A}>\mathrm{G}}{\mathrm{c} . \text { (Thr209Ala }}$
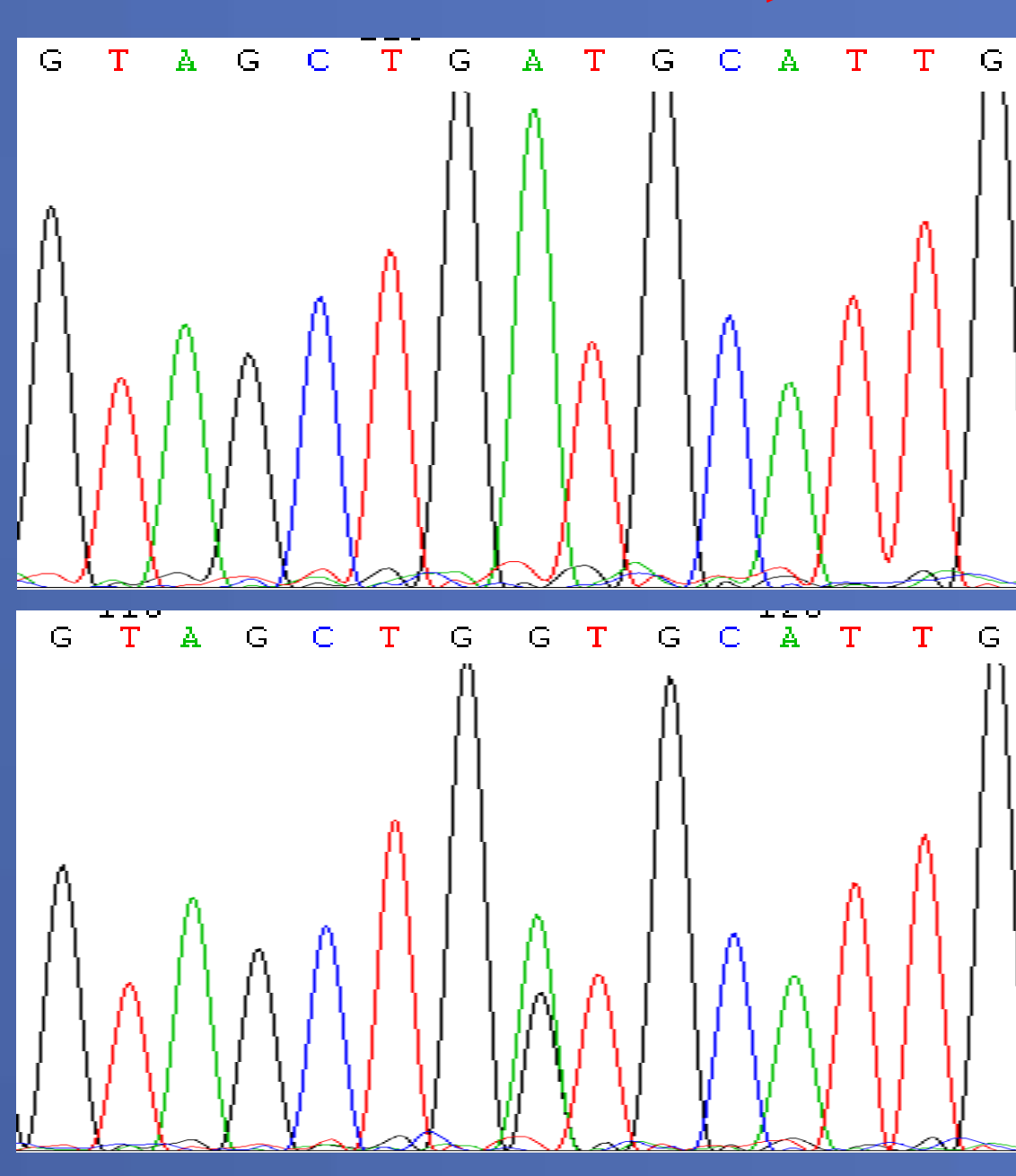

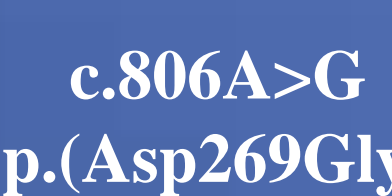

Figure 1 - PRKAR1A mutations detected by Sanger sequencing.

The upper panel shows the representative structure of PRKARIA (protein domains and gDNA) and the genomic are the electropherograms of missense mutations found in our series and of wild-type reference sequences.
Multiplex Ligation-dependent Probe Amplification assay (MS-MLPA)

Dosage of allele segments was performed by MLPA using the SALSA MLPA P264 Human Telomere-9 probemix (MRCHolland, Amsterdam, The Netherlands). The protocol was implemented following the manufacturer's recommendations. MLPA PCR products were separated by capillary electrophoresis using ABI3130xI Genetic Analyzers (Perkin-Elmer Corp.) with an internal size standard GeneScan 500 LIZ (Applied Biosystems, Foster City, CA. Data analysis was performed using GeneMapper software (Applied Biosystems, Foster City, CA) and Coffalyser v9.4 (MRC-Holland, Amsterdam, The Netherlands).

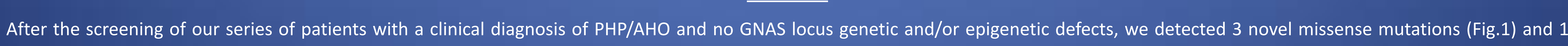

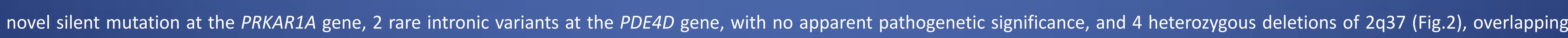
with previously described rearrangements affecting this subtelomeric region.

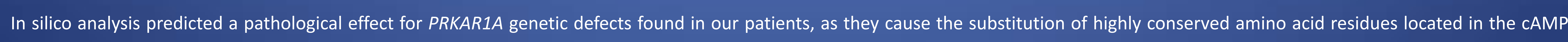

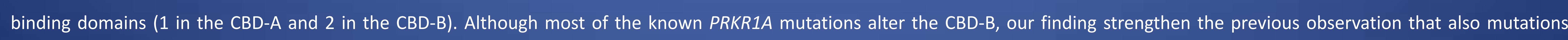

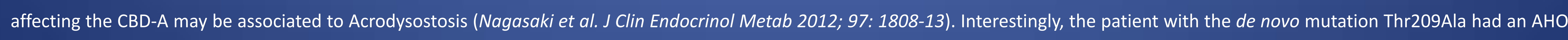

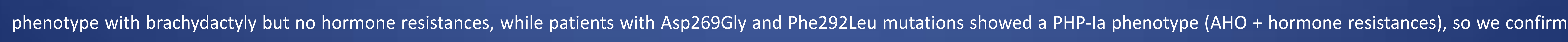
the variability of the clinical phenotype caused by PRKRA1A mutations.

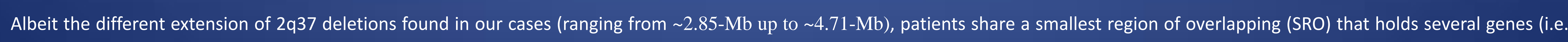

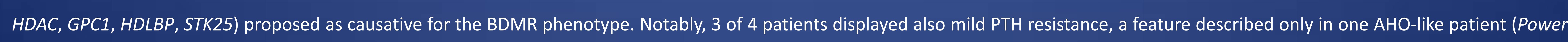
et al. J Med Genet 1997; 34: 287-290), but we exclude RCDI as candidate gene as its location is upstream the deleted region found in our series.

\section{CONCLUSIONS}

Our data further confirm the molecular and clinical overlap among these disorders and highlight the complexity in performing an accurate diagnosis of PHP, as well as the pivotal role of the CAMP pathway in the development of the AHO phenotype. 